\title{
Characterization of the regulation mechanism of grapevine microRNA172 family members during flower development
}

Xin Sun ${ }^{1,2+}$, Mengqi Wang ${ }^{2 \dagger}$, Xiangpeng Leng ${ }^{1,3}$, Kekun Zhang ${ }^{2}$, Gengsen Liu ${ }^{1,3^{*}}$ and Jinggui Fang ${ }^{1,2,3^{*}}$

\begin{abstract}
Background: Grapevine (Vitis vinifera L.), which has important nutritional values and health benefits, is one of the most economically important fruit crops cultivated worldwide. Several studies showed a large number of microRNAs (VvmiRNAs) involved in the modulation of grape growth and development, and many VvmiRNA families have multiple members. However, the way by which various members from the same miRNA family work is unclear, particularly in grapes.

Results: In this study, an important conserved VvmiR172 family (VvmiR172s) and their targets were set as a good example for elucidating the interaction degree, mechanism, and spatio-temporal traits of diverse members from the same miRNA family. miR-RACE and Stem-loop RT-PCR were used to identify the spatio-temporal expressions of various members of VvmiR172s; together with RLM-RACE, PPM-RACE, Western blot, transgenic technologies, their interaction degree, and regulation mechanism were further validated. The expression of VvmiR172c was significantly higher than that of VvmiR172a, $b$, and $d$ and showed a positive correlation with the abundance of VVAP2 cleavage products. These findings indicated that VvmiR172C might be one of the main action factors of the VvmiR172 family in flower development. The ability of $\mathrm{VvmiR172c}$ to cleave target genes differed due to divergence in complementary degree with VVAP2 and expression levels of various members. In VvmiR172 transgenic lines, we observed that 35S::VvmiR172C resulted in the earliest and abundant flowering, indicating the strong function of VvmiR172c. In contrast, the nonsignificant phenotypic changes were detected in the VVAP2 transgenic lines. The qRT-PCR and Western bolt results demonstrated that VvmiR172C plays a major role in targeting VVAP2.

Conclusions: VvmiR172 up-regulated the expression of NtFT and decreased the expression of NtFLC. The up/down regulation of $V_{v}$ miR172c was the most pronounced. The functions of four VvmiR172 members in grape differed, and miR172c had the strongest regulation on AP2.
\end{abstract}

Keywords: Grapevine, VvmiR172 family members, Regulation mechanism, VVAP2, Flower

\footnotetext{
*Correspondence: gsliu@qau.edu.cn; fanggg@njau.edu.cn

${ }^{+}$Xin Sun and Mengqi Wang contributed equally to this work.

'College of Horticulture, Qingdao Agricultural University, Qingdao 266109,

China

Full list of author information is available at the end of the article
}

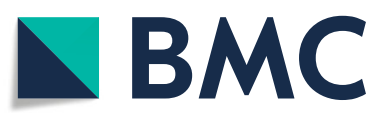

C C The Author(s). 2020 Open Access This article is licensed under a Creative Commons Attribution 4.0 International License, which permits use, sharing, adaptation, distribution and reproduction in any medium or format, as long as you give appropriate credit to the original author(s) and the source, provide a link to the Creative Commons licence, and indicate if changes were made. The images or other third party material in this article are included in the article's Creative Commons licence, unless indicated otherwise in a credit line to the material. If material is not included in the article's Creative Commons licence and your intended use is not permitted by statutory regulation or exceeds the permitted use, you will need to obtain permission directly from the copyright holder. To view a copy of this licence, visit http://creativecommons.org/licenses/by/4.0/ The Creative Commons Public Domain Dedication waiver (http://creativecommons.org/publicdomain/zero/1.0/) applies to the data made available in this article, unless otherwise stated in a credit line to the data. 


\section{Background}

In plants, miRNAs play important roles in a number of physiological processes, such as growth development and biotic and abiotic stress responses [1-5]; they can regulate the expression levels of their target genes by cleavage. Other miRNAs emerged as promising approaches for plant breeding applications, such as crop improvement strategies [6, 7].

Many plant miRNAs have been identified [8-10], but the majority of these miRNAs remain poorly characterized. Speedy changes in miRNA expression profiles might contribute to plant functions [11-16], and such changes have been described in allotetraploid cotton (Gossypium hirsutum L.) [17]. Also, many miRNAs are largely conserved and belong to multigene families [18]. These differences in mature sequences could lead to varieties of target genes type or change the cleavage levels. Multigene families are predicted to target the same or overlapping sets of genes, indicating the possibility of substantial functional redundancy among miRNAs. Other researchers showed miRNA family members that play a specific role in regulation [19-21]. miR156c can participates in the stress tolerance process by regulating its downstream genes, SPL9 and DFR, which take part in this process by influencing the metabolism of anthocyanin [21]. All these abovementioned studies indicated that the action of a full miRNA family was not comprehensively and systematically investigated, and thus, it was difficult to gain insight into the roles of miRNA family.

In grapes, although several studies had identified a large number of VvmiRNAs [16, 22-24], these works focused on the identification of VvmiRNA sequences and their expression profiles at some special tissues/organs. Few studies report research on one miRNA family. Our previous studies revealed that the various members of same VvmiRNA family possessed differential expression profiles and showed the dynamic variation of their expression levels during the grapevine development process [16]. The divergence on the complementary degree between various members of VvmiRNA family and their targets might affect the action degree and regulation mechanism of VvmiRNAs on their target genes. However, the difference in the sequences of various members from the same VvmiRNA family might be an important factor influencing the cleavage degree of miRNAs on their target genes. The differential expression profiles of various members might be another factor affecting interaction. So far, studies that systematically investigated the action degree and regulation mechanism of various members from the same miRNA family on their target genes are lacking. Most previous studies focused on the roles of all miRNA family. Therefore, to more comprehensively recognize the roles of a miRNA family with multiple members, it is necessary and significant to carry out a systematic analysis of each member of a VvmiRNA.

In the previous studies, the experimental validation of miRNAs focused on determining their expressions by Northern blotting and/or RT-PCR. Interestingly, although these two technologies could demonstrate the existence and size of miRNAs, they did not determine their full precise sequences [23-26]. In particular, for miRNA family with multiple members, just the mixture of multiple members' expressions was detected instead of those of each member. As described above, the variation of diverse members' sequences from the same miRNA family might affect their complementary degree with corresponding target genes. Thus, these variations might be the action degree and regulation mechanism of miRNAs on their target genes. We developed an integrative strategy of combining miR-RACE and stem-loop RT-PCR together with RLM-RACE, PPM-RACE, and Western blot that could accurately determine the full precise sequences of each member from the same miRNA family. This strategy would also detect the expression.

Levels and cleavage products of each member from the same miRNA family to exactly identify the action degree and regulation mechanism of each member.

The functional exploration of individual miRNA members was meaningful for understanding the function of miRNAs. To discover the regulation mechanism and function of various members from the same miRNA family, VvmiR172 family was used as a typical case investigation in this study. miR172 is an important family that plays critical roles in flower development; it affects the flowering time period, floral organ attributes, and the floral identity by regulating the expression of AP2-like genes [27-29]. Currently, several studies showed that most grapevine miRNAs belong to a multigene family, and several members are only different in one to several nucleotides $[8,16$, 23, 24]. It is now important to describe the VvmiRNA family members to better understand how miRNAs contribute to grapevine development.

In plants, many miRNAs belong to some multigene families, in which the members are different in mature sequences from individual or multipe nucleotides [18]. The differences in sequences cause variations in matching levels with target genes. Various family members have different temporal and spatial expression characteristics. These differences in mature sequences could lead to varieties of target gene type or change the cleavage levels. Many previous reports were conducted on one member; however, these studies indicate only the characteristics of expression and transgene function [22, 30, 31]. Little attention has been paid to the function and strength of other miRNA family members. Most studies focus on the regulatory function of the whole miRNA 
family [32-34]. These works did not study each family member. In the present study, miR-RACE, stem-loop RT-PCR, RLM-RACE, PPM-RACE, and transgenic technologies were used to investigate the functions of four miR172 members and the target gene of AP2-likegenes. This work can elucidate the expression and regulation mechanism on target genes of miRNA family members during grapevine development and provide an efficient strategy that can be used to uncover the role of the different miRNA family members.

\section{Result}

\section{Diversity of members from miRNA family and their matching degree with targets}

Recently, high-throughput sequencing analysis based on small RNA sequencing data suggested that many miRNA families are not a single sequence. A miRNA family is composed of a series of miRNAs with various lengths/ sequences. In this study, VvmiR172 family has four members, and sequences of all four members were different (Fig. 1). The alignment analysis of four VvmiRNA family members revealed an overall well-conserved consensus with few variations. The miRNA family has multiple members, and its sequence mostly exists at one or more base differences at the $5^{\prime}$ or $3^{\prime}$ end. The difference of miRNA sequence could lead to the change of complementation with target gene. Among four members, VvmiR172c possessed the least mismatch bases with target gene VvAP2, followed by VvmiR172d, VvmiR172b, and VvmiR172a (Fig. 1). Minor variation in the bases of miRNA sequence may alter its ability to regulate target mRNAs.

\section{Dynamic changes in various members of miRNA family}

\section{during grapevine flower development}

There are many members in the same miRNA family in grapevine that may result in functional redundancy or in a function that has spatial-temporal specificity, but the individual contributions are still unknown. Thus, we examined whether each member plays a particular function in flower development. The miR-RACE and stem- loop RT-PCR was used to analyze the expression levels of different members in the same VvmiRNA family. The expression trends between different VvmiR172 members showed specificity in flower development (Fig. 2a, b, c). However, the proportion of miR172b and $c$ in total expression is relatively higher than the others (Fig. 2a, b); this finding is similar to our previous high throughput results [16]. The trend of VvmiR172c expression was consistent with that of the VvmiR172 family, but the expression of VvmiR172b was downregulated during flower development (Fig. 2a, b, c), thereby indicating that diverse members of VvmiR172 family might possess various regulation mechanisms during grape flower development, and VvmiR172b and $\mathrm{c}$ might play the key roles in this process.

\section{The role of individual miRNA members in regulating target gene expression}

Plant miRNAs control gene expression post-transcriptionally through miRNA-mediated cleavage. The spatio-temporal expression profiles of target genes can provide important information in the growth and development of grapevine. Verifying which miRNA members are working on a target and when this event occurs is important to better understand the roles of miRNAs in flower development.

Considering the fact that the individual members of VvmiR172 family showed different transcription by Stem-loop RT-PCR and miR-RACE, we analyzed the roles of each VvmiR172 family members in regulating the target's expression. The authenticity of the target genes $V v A P 2$, for the VvmiR172 family, has been validated $[8,16]$. Here we measured the abundance of multiple miRNA members/target mRNA pairs. We found that these cleavage fragments change during flower.

The transcript level of VvmiR172 increased from 9DBF to 5DBF/7DBF and then decreased (Fig. 2a, b, c). The proportion of VvmiR172b and c expression had certain advantages (Fig. 2a, b). VvAP2 is a typical target gene for VvmiR172 that is involved in regulating flower development. In addition to the expression of VvmiR172b, the expressions of the remaining three

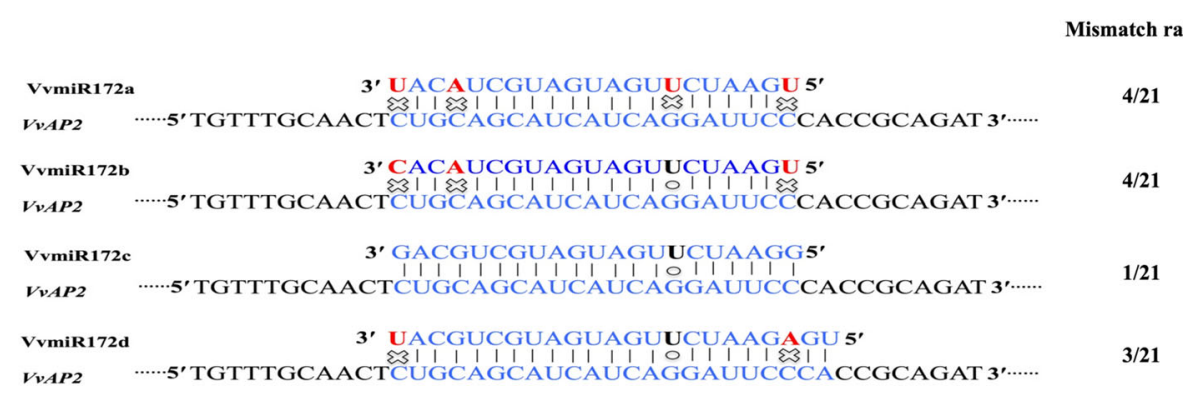

Fig. 1 Conserved sequence variation in mature VvmiR172 members and the match between members of VvmiR172 and target genes. WatsonCrick pairing (vertical dashes), mismatch (fork and circles) and G: $U$ wobble pairing (circles) is indicated 


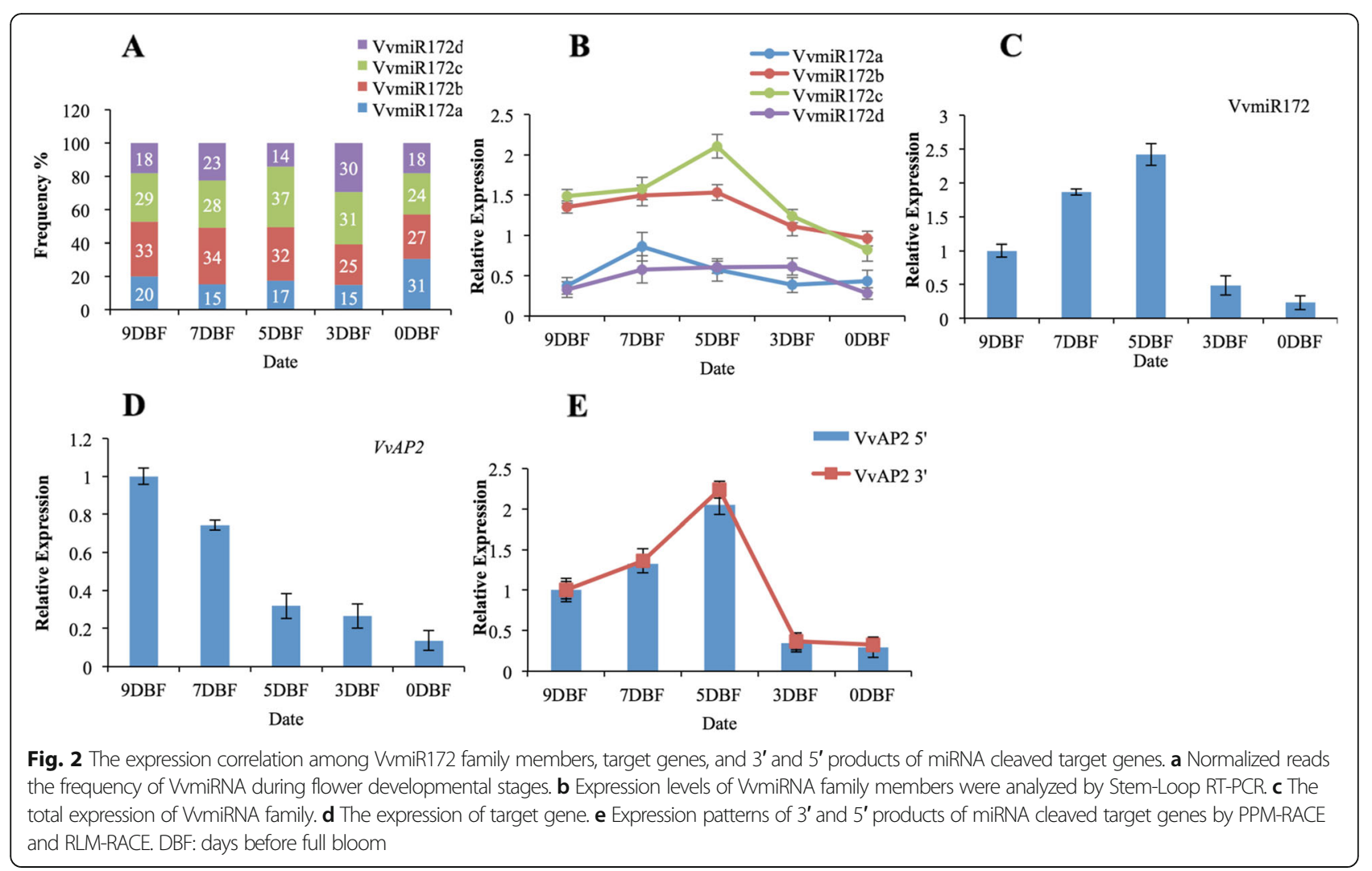

VvmiR172 members were positively correlated with the yield of $V v A P 2$ cleavage products (Fig. 2d, e). This phenomenon could be attributed to the fact that VvmiR172a, c, and d co-participated or only one VvmiR172 member participated in the regulation of $V v A P 2$ expression (Fig. 2d). To gain further insight into the regulation mechanism of VvmiR172, additional transgenic research is carried out to explore the VvmiR172 family-specific function. In addition, the four members of VvmiR172 family were easily distinguished. Variable control in transgenic detection analysis was probable.

\section{Functional analysis of each Vvmi172 family members in Nicotiana tabacum}

To determine the regulation patterns of target gene AP2 by each VvmiR172 family member, we constructed four over-expression vectors of VvmiR172 family members and transformed them into tobacco ( $N$. tabacum). The accumulation of VvmiR172 in tobacco transgenic lines was analyzed by Stem-loop RT-PCR and miR-RACE. Each VvmiR172 member was detectable in 35S:: VvmiR172 lines transformed tobacco (Fig. 3).

The function of miR172 was related to the formation of floral patterning $[28,29]$. Interestingly, ectopic expression of each VvmiR172 member advanced flowering time (Fig. 4). Floral patterning defects, including sepal- to-petal, was not observed. 35S::VvmiR172c lines comparable with other members' transgenic lines and wildtype (WT) showed early flowering, and the numbers of nods increased. VvmiR172c leaves were wrinkled, and paraffin sections revealed abnormal vascular tissue cells. The plant height of VvmiR172b, c, and d increased obviously compared with the WT. However, VvmiR172c had higher plant height compared with VvmiR172b and $\mathrm{d}$. Through the phenotypic observation of transgenic tobacco, we can judge that VvmiR172c played an important role in the development of flower and other phenotypic characters of the plant. Interestingly, there were no obvious phenotypic changes in VvAP2 transgenic plants (Fig. 4).

To further determine the regulatory role of each VvmiR172 family member, the abundance of tobacco AP2 (NtAP2) degradation fragments in individual VvmiR172 members' transgenic lines was investigated (Fig. 5). The expression of transgenic lines' cleavage products were higher than those of WT. Although the expression of NtAP2 degradation products in the other three VvmiR172 member transgenic lines were higher than those of WT but still lower than those of 35S:: VvmiR172c. The highest cleaved products expression was recorded for 35S::VvmiR172c in all four transgenic lines (Fig. 5a). The NtAP2 degradation fragments of 35S::VvmiR172a had weak expression in all VvmiR172 

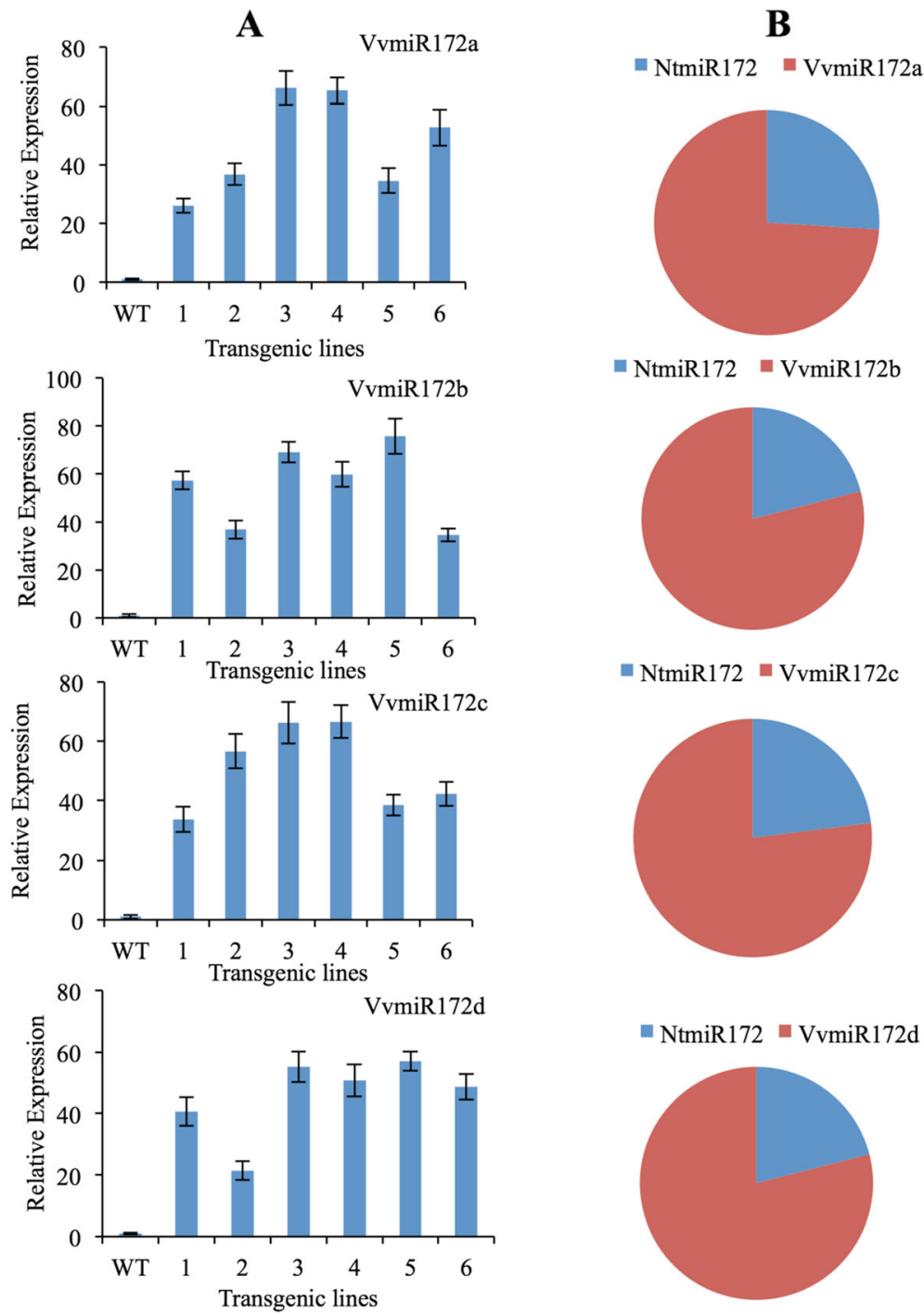

Fig. 3 qRT-PCR (a) and miR-RACE (b) analysis of each VvmiRNA family member levels in transgenic and WT tobaccos. b In transgenic plants, the 5 'miR-RACE and 3'miR-RACE products were sequenced. The frequency of each member VvmiR172 sequence was statistically analyzed as its percentage in the total miR172 sequences of all the PCR-amplified. The frequencies of all the miR172, considered as their relative expression levels. The red block showed the expression frequencies of VvmiR72 members. Blue Block showed the expression frequencies of NtmiR172

transgenic lines. In addition, NtAP2 protein accumulated at a lower level in the each of the four VvmiR172 transgenic lines (Fig. 5b). These results proved that VvmiR172c plays a key role in regulating AP2. The transgenic plants had early flowering phenotypes. The transcription levels of some genes related to floral development were detected in transgenic tobacco. The NtFT expression in all four VvmiR172 members transgenic tobacco was higher than that of WT tobacco, and the expression of NtFLC was lower than that of WT tobacco (Fig. 6). Similarly, the highest expression of NtFT was found in 35S::VvmiR172c. However, the difference in NtFLC expression between four VvmiR172 members transgenic was not obvious (Fig. 6). Each VvmiR172 member had different functions in flower development, and VvmiR172c plays an important role in regulating AP2 transcription.

\section{Discussion}

miRNAs function broadly to regulate many aspects of plant development, including flower development, from floral induction to floral organ specification. However, miRNAs belong to a multigene family, and the difference of various members from the same miRNA family only exists in few different nucleotides or single nucleotide in sequences. In model plants, some reports suggested that members of different miRNA families have different levels of expression and have differences or redundancies 


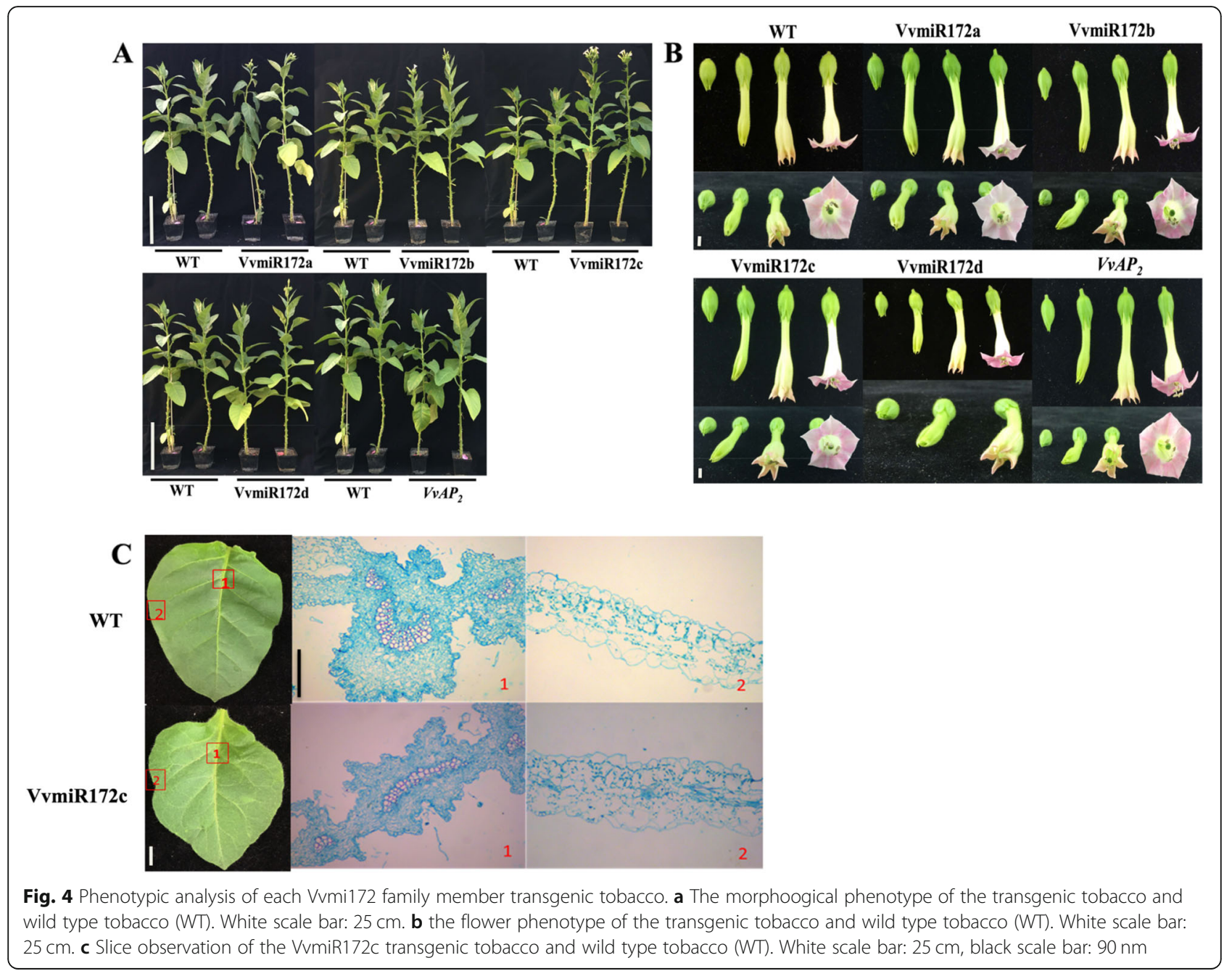

in their functions. For example, the study of all three miR164 family members mutation indicated that miR164c contributed to a larger extent to the control of shoot development than its two sister miRNAs [19].

In the present study, we observed highly dynamic changes of miRNAs and their family members by StemLoop RT-PCR and miR-RACE in grapevine (Fig. 2a, b, c). The expression patterns of VvmiRNA exhibited a certain degree of inverse relationship in expression as expected for target genes, thereby suggesting that these genes might be actively cleaved by VvmiRNA. The expression of VvmiRNA family is not the same as that of any members of the family. There is a positive correlation between the expression of degradation products and the change of miRNA expression, which can be regarded as a standard in evaluating the function of miRNA [35]. The spatio-temporal of different miRNA members could determine the spatio-temporal of target genes. The expression of VvmiR172c/b was higher than that of VvmiR172ad and showed a positive correlation with the abundance of cleavage products, which indicated that VvmiR172c played an important role in flower development (Fig. 2a, b, c). Other reports also suggested that miR172c was an important development regulator $[29,36]$. Based on our findings, we propose that different members of the same VvmiRNA miRNA family perform different tasks during grapevine development, with certain key members acting as the main regulators. The abundance of VvmiR172c was the highest, and its ability to regulate $V v A P 2$ had a great advantage. It had a stronger function of cracking AP2 mRNA. This shows a positive correlation between the expression level of miRNA and its ability to cleave mRNA. Interestingly, the miRNA members with significant regulation had a strong function of cracking AP2 mRNA low mismatching rate with target genes (Fig. 1). AtmiR172 can repress AtAP2 at the level of translation [37]. However, The AtmiR172 have higher mismatches with AtAP2, VvmiR172 had lower mismatching rate with $V v A P 2$. In previous reports, miR172 possessed the least mismatch bases with target $A P 2$, and it could regulate the root and flower development through miR172-induced AP2 

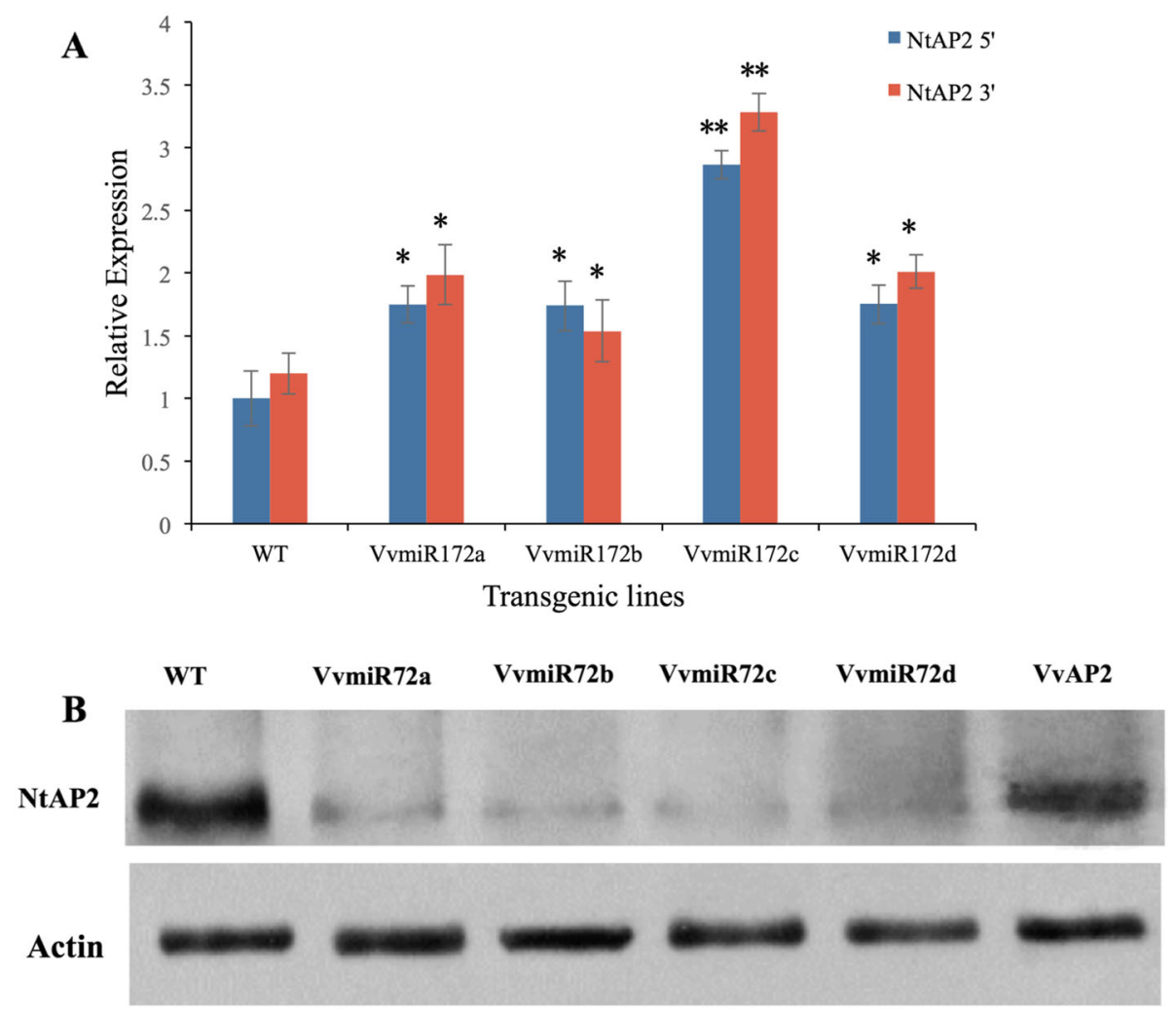

Fig. 5 Expression patterns of the cleaved NtAP2 products and NtAP2 protein levels in transgenic and WT tobacco. a Expression patterns of $3^{\prime}$ and 5' products of each VvmiR172 members cleaved target genes from transgenic and WT tobacco by RLM-RACE and PPM-RACE. b Immunoblot analysis of NtAP2 protein levels in transgenic and WT tobaccos. Values are the mean of three replicates, and differences with a $P$-value $<0.05$ were considered significant. ${ }^{*} P<0.01 ;{ }^{*} P<0.001$

cleavage [36, 38, 39]. VvmiR172c had only one error rate with the target gene $V v A P 2$, whereas the other three had three or four mismatches with the target gene. The mismatch between miRNA and target genes was low, and the ability to degrade target genes may be stronger.

Since the sequences of four members of the VvmiR172 family differ, they can be distinguished, and the expression of each member in flowers is not obvious. Most importantly, the VvmiR172 matches the tobacco and grape AP2 exactly the same.

The regulation of target genes by VvmiR172 family members was further investigated by transgenic tobacco. miR172 regulates flowering time and floral organ formation via the degradation and inhibition of $A P 2$ expression [27-29]. Over-expression of miR172 can lead to early flowering (Table 1, Fig. 4). The observation of tobacco's ectopic expression of four members of the miR172 family showed early flowering and the increase in the flowering quantity. However, the phenotype of floral organ formation has not been observed. This finding may be because the inhibitory effect of VvmiR172 on $N t A P 2$ is weaker than that of miR172 in tobacco. Through the transgenic phenotype, we can see that 35S::
VvmiR172c had the earliest flowering time and the largest amount of flowering (Fig. 4), which shows that VvmiR172c had the strongest function.

miRNAs have been heavily studied in grapevine, but VvmiRNAs and their family members are not thoroughly described. We also analyzed the abundance of AP2 degradation products and AP2 protein accumulated in transgenic tobacco plants (Fig. 5). VvmiR172c had the largest cleavage effect on NtAP2. No significant phenotypic changes were observed in $V v A P 2$ transgenic plants. We propose that $A P 2$ alone cannot play a regulatory role, and over a certain range, its over-expression did not affect the phenotype of plants. The disruption of AP2 caused its function to weaken, and the phenotype caused abnormal flowering time. miR172 could down regulate target genes with flowering inhibitory activity (e.g. TOE1, SMZ, and others), which eventually converged to FT, thereby leading to early blooming [40]. $F L C$ can inhibit flowering by weakening the expressions of $F T$ and SOC1 [40, 41].

Based on our data, members of a single family likely perform different tasks during grapevine development. We provide evidence that the expression levels and 

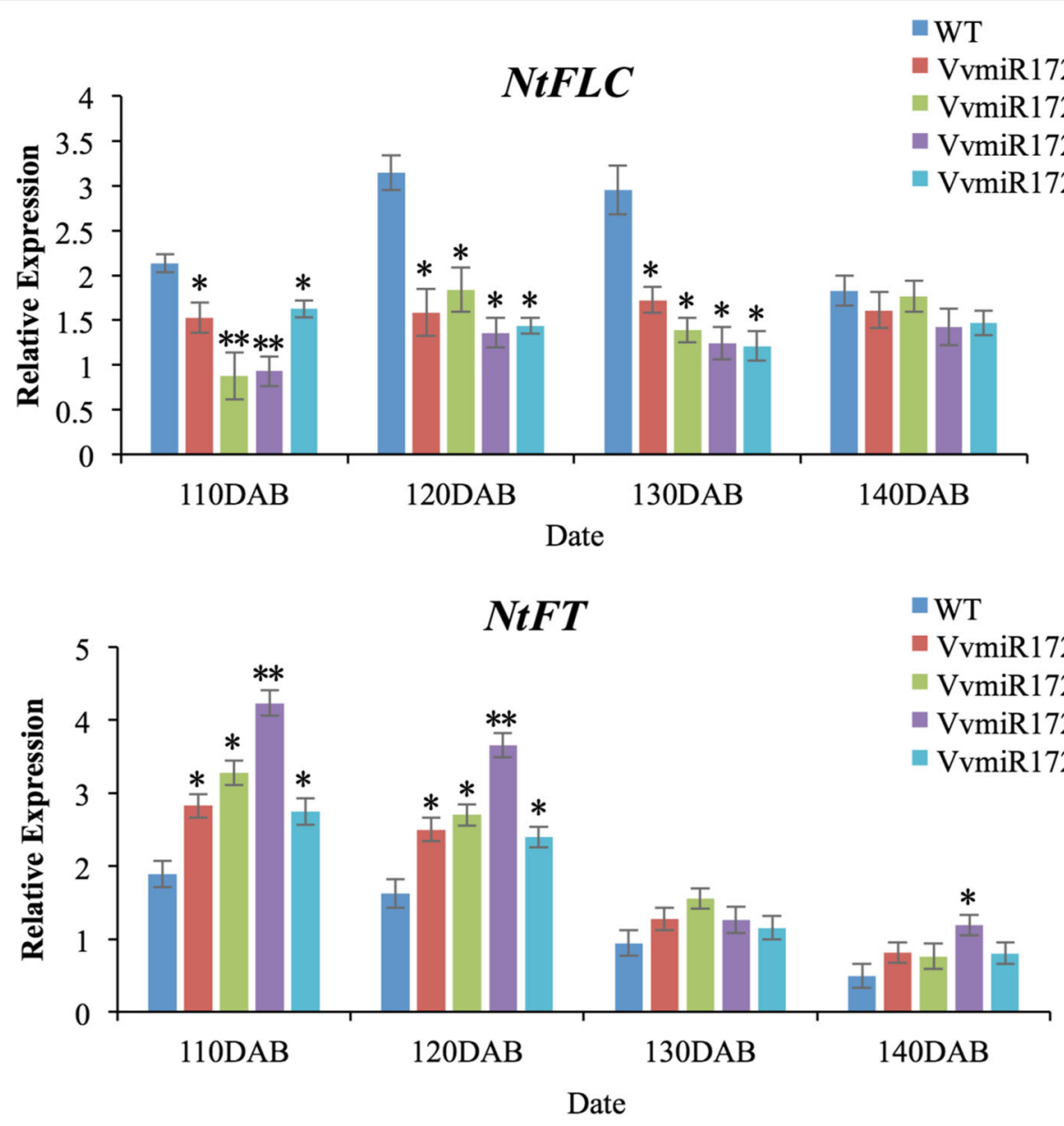

Fig. 6 Expression analysis of genes related to flower development (NtFLC and NtFT) in transgenic and WT tobacco. DAB: days after breeding. Values are the mean of three replicates, and differences with a $P$-value $<0.05$ were considered significant. ${ }^{*} P<0.01$; ${ }^{* *} P<0.001$

Table 1 Comparison of physiological index between transformed and non-transformed plantlets

\begin{tabular}{|c|c|c|c|c|c|c|c|c|c|}
\hline & $\begin{array}{l}\text { Height } \\
(\mathrm{cm})\end{array}$ & $\begin{array}{l}\text { Stemdiameter } \\
(\mathrm{cm})\end{array}$ & Node number & $\begin{array}{l}\text { Internode } \\
\text { length }(\mathrm{cm})\end{array}$ & $\begin{array}{l}\text { Ratio of length } \\
\text { to width }\end{array}$ & $\begin{array}{l}\text { Flowering } \\
\text { time (day) }\end{array}$ & $\begin{array}{l}\text { Flowering } \\
\text { amount }\end{array}$ & $\begin{array}{l}\text { Flower } \\
\text { diameter } \\
(\mathrm{cm})\end{array}$ & $\begin{array}{l}\text { Flower } \\
\text { length }(\mathrm{cm})\end{array}$ \\
\hline WT & $\begin{array}{l}78.36 \pm \\
1.6253\end{array}$ & $1.03 \pm 0.0951$ & $38.33 \pm 0.5774$ & $2.74 \pm 0.5177$ & $1.91 \pm 0.0904$ & $135.2 \pm 6.2$ & $71.3 \pm 9.07$ & $2.14 \pm 0.0547$ & $4.64 \pm 0.1141$ \\
\hline VvmiR172a & $\begin{array}{l}79.86 \pm \\
1.0525\end{array}$ & $1.06 \pm 0.1103$ & $37.66 \pm 1.5275$ & $2.96 \pm 0.3911$ & $1.84 \pm 0.0485$ & $126.3 \pm 5.6^{*}$ & $68.3 \pm 8.51$ & $2.13 \pm 0.1141$ & $4.74 \pm 0.1517$ \\
\hline VvmiR172b & $\begin{array}{l}83.78 \pm \\
0.8083^{*}\end{array}$ & $1.12 \pm 0.1456$ & $38.67 \pm 0.5884$ & $2.82 \pm 0.2588$ & $1.89 \pm 0.0955$ & $122.8 \pm 5.2^{*}$ & $76.2 \pm 6.08^{*}$ & $2.11 \pm 0.0512$ & $4.61 \pm 0.2121$ \\
\hline VvmiR172c & $\begin{array}{l}93.43+ \\
1.3283^{* *}\end{array}$ & $1.11 \pm 0.1731$ & $43.12 \pm 1.2358^{*}$ & $3.02 \pm 0.2168$ & $2.04 \pm 0.0702$ & $118.3 \pm 3.6^{* *}$ & $87.3 \pm 4.35^{*}$ & $2.08 \pm 0.0472$ & $4.56 \pm 0.1673$ \\
\hline VvmiR172d & $\begin{array}{l}81.73 \pm \\
2.2723^{*}\end{array}$ & $1.07 \pm 0.1723$ & $37.33 \pm 1.1547$ & $3.04 \pm 0.2511$ & $1.95 \pm 0.0874$ & $128.4 \pm 4.8^{*}$ & $66.4 \pm 8.62$ & $2.02 \pm 0.0836$ & $4.58 \pm 0.1924$ \\
\hline VvAP2 & $\begin{array}{l}76.84 \pm \\
1.3645\end{array}$ & $1.01 \pm 0.2061$ & $38.67 \pm 1.3425$ & $2.87 \pm 0.3742$ & $1.86 \pm 0.0531$ & $143.8 \pm 6.5$ & $63.7 \pm 7.23$ & $2.11 \pm 0.0701$ & $4.62 \pm 0.0837$ \\
\hline
\end{tabular}


ability to degrade the target genes of miRNA members within the same family vary in grapevine. From this, we infer that complex mechanisms regulate the expression of these members.

Based on our findings, we propose that the roles of miRNAs and their families' members extends beyond their initial expression and continues to be active in subsequent developmental stages. We also report great differences in the expression levels of miRNA members (Fig. 7). This work contributes to our understanding of the regulatory network underlying grapevine development.

\section{Conclusions}

VvmiR172 up-regulated the expression of NtFT and decreased the expression of NtFLC (Fig. 6). The up/down regulation of VvmiR172c was most pronounced. All of the above results showed that the function of four VvmiR172 members in grape differed, and miR172c had the strongest regulation on $A P 2$.

\section{Methods}

\section{Plant material}

Plant tissue was collected at each stage of flower development. Tissue was collected from 6-year-old table grapevine 'Wink' (V.vinifera) grown under standard conditions. The grapevines used in this study were from the Fruit Experimental Farm, Nanjing Agricultural University, Nanjing, China. After collection, samples were frozen in liquid nitrogen and stored at $-80^{\circ} \mathrm{C}$ until use. The Nicotiana benthamiana was obtain from Laboratory of genetic breeding and genomics of fruit trees (NJAU). All Plant materials were provided free of charge, and were not endangered materials or species.

\section{RNA isolation, and CDNA synthesis}

RNA was isolated from grapevine plant tissue using standard molecular biology protocols and following manufacturer's instructions, as previously described $[5]$.

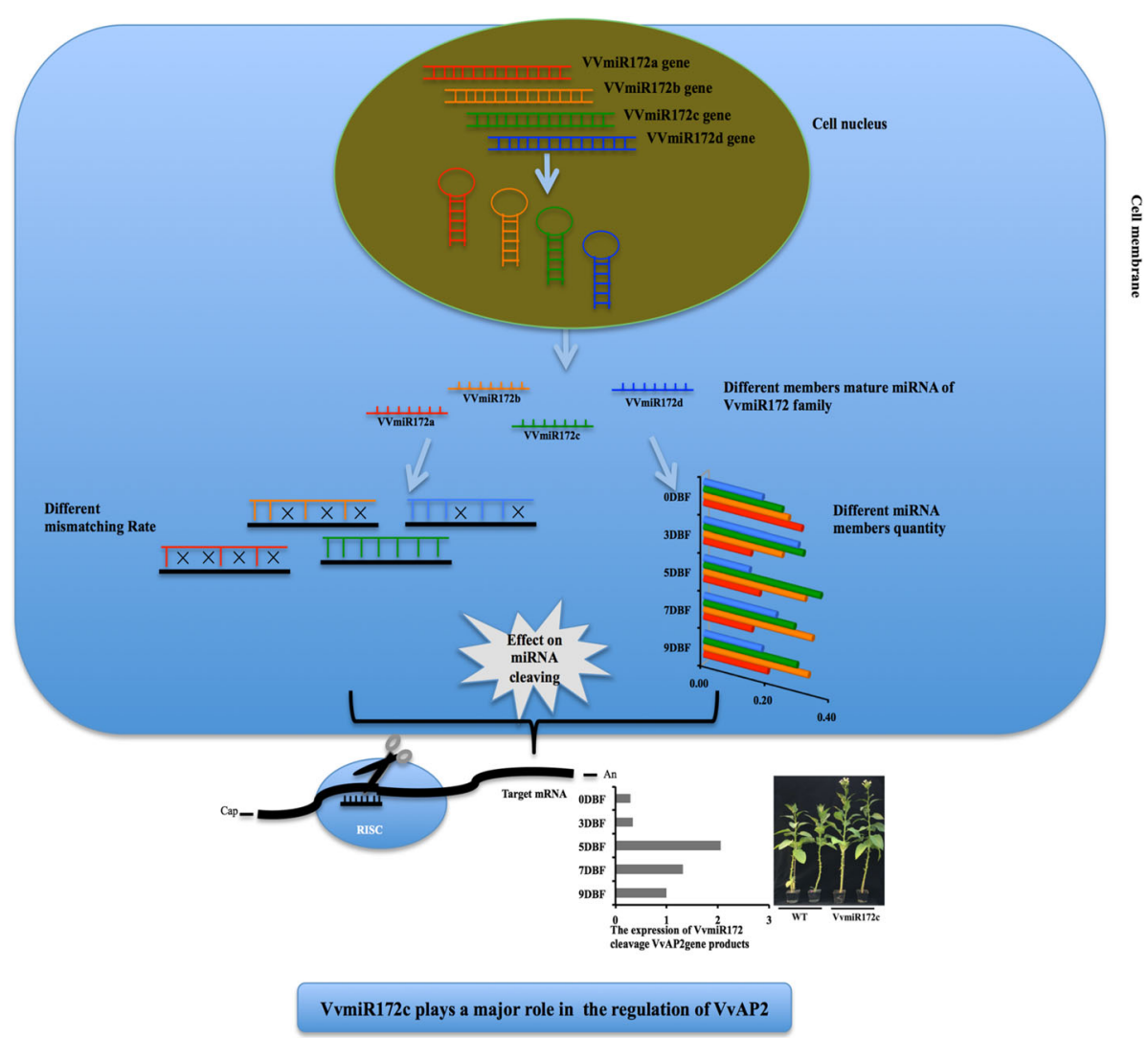

Fig. 7 The effect on the cleaving of different miRNA members of the same family. Their expression of VvmiR172 members had great differences, the diversity in sequences and expression levels lead to miRNA regulated target gene VVAP2 ability difference. The mismatch between VvmiR172 and target gene VVAP2 was low, and the ability to degrade target genes may be stronger. Red, yellow, green, blue and black lines indicated VvmiR172a, VvmiR172b, VvmiR172c and VvmiR172d and VvAP2, respectively. The black fork indicated the mismatch between VvmiR172 members with target gene VVAP2 


\section{Verification of microRNA expression patterns}

First, non-gene-specific products were amplified as previously described, with minor modifications [25]. The 5' RACE and 3' RACE clones were sequenced [42], and the relative abundance of each VvmiRNA sequence was analyzed. The results were expressed as relative expression.

\section{Quantitative stem-loop qRT-PCR of mature miRNA}

Grape flower RNA $(1 \mu \mathrm{g})$ from was reverse transcribed using miRNA-specific reverse stem-loop transcription primers and normalized to Action (Supplemental Table S1). Relative expression levels were calculated using the $2^{-\triangle \triangle C T}$ method and three biological replicates.

Real-time PCR of total expression of VvmiR172 and AP2 PolyA tailing assay was used to detect the expression of VvmiR172 [43]. Our miRNA-enriched library was used as the template for RT-PCR. To amplify VvmiR172 from reverse transcribed cDNAs, we used the part of the VvmiR172 sequence (GAATCTTGATGATGCTACA) as the forward primer and the R16328 (ATTCTAGAGG CCGAGGCGGCCGACATG) as the reverse primer [23]. The expression of mature miRNA was normalized to that of 5.8S rRNA. The expression of the VvmiR172 target gene AP2 was done as previously described (Supplementary Table S1) [23].

Relative expression levels were calculated using the comparative $\mathrm{Ct}$ method with normalization of data to the geometric average of the reference genes by the $2^{-\triangle \Delta \mathrm{CT}}$ method.

\section{Prediction of targets gene of VvmiR172}

miRNAs bind to the protein coding region of their mRNA targets with perfect or near-perfect sequence complementarily. The strategy of VvmiR172 targets prediction was according to the methods reported by Song et al. [25]. These criteria included allowance for no more than two mismatches in the region complementary to nucleotide 1-9 from the 5'end of miRNA, no mismatch for positions 10 and 11, and another two mismatches allowed between positions 12 and 21/24. The number of allowed mismatches at complementary sites between miRNA sequences and potential mRNA targets was no more than four, and no gaps were allowed at the complementary sites.

\section{A novel RLM-RACE plus PPM-RACE with qRT-PCR cleavage production method}

We developed a novel technique for the validation of miRNA target mRNAs that has six steps: (1) library preparation (2), nested RLM-RACE and PPM-RACE, (3) amplicon cloning and sequencing, (4) slicing site detection, (5) qPCR of miRNA-cleaved target mRNA, and
(6) cloning and sequencing of products for validation $[25,44]$.

\section{Plant transformation and growth conditions}

Tobacco (Nicotianatabacum) leaf explants were transformed using the A. tumefaciens method [45]. The plants were regenerated at $24^{\circ} \mathrm{C}$ under $16 \mathrm{~h}$-light $/ 8 \mathrm{~h}$ dark cycles and transformants identified by kanamycin resistance. After root induction, plants were transferred to soil and grown at $25^{\circ} \mathrm{C}$.

\section{Protein extraction and immunoblotting}

Protein wasextracted from leafs of 60 days transgenic tobacco and WT under standard long-day ( $16 \mathrm{~h}$ of light and $8 \mathrm{~h}$ of darkness) conditions. The isolation of protein was made by homogenization in $50 \mathrm{mMTris}-\mathrm{HCl}, \mathrm{pH}$ 8.0, $10 \mathrm{mMNaCl}, 0.1 \mathrm{M}$ PMSF, and $0.1 \mathrm{M}$ DTT, with subsequent centrifugation at $13,000 \mathrm{~g}$ for $30 \mathrm{~min}$ at $4{ }^{\circ} \mathrm{C}$. After electroblotting on a nitrocellulose membrane, protein gel blot analysis was performed using antibodies against NtAP2. Plant Actin was used as loading control.

\section{Supplementary information}

Supplementary information accompanies this paper at https://doi.org/10. 1186/s12870-020-02627-6.

Additional file 1: Table S1. Sequences of primer and use.

Additional file 2. The orginal figure of western blot.

Additional file 3. The coding sequences of VVAP2 and NtAP2.

Additional file 4. The sequences of VvmiR172 family members.

Abbreviations

miRNA: MicroRNA; AP2: APET ALA2; RLM-RACE: RNA ligase-mediated 5' rapid amplification of CDNA ends; PPM-RACE: Poly (A) polymerase-mediated 3' rapid amplification of CDNA ends; miR-RACE: miRNA-rapid amplification of CDNA ends; RT-PCR: Reverse transcription-polymerase chain reaction; qRTPCR: Quantitative real-time PCR; RT-PCR: Reverse transcription PCR

\section{Acknowledgements}

We thank Dr. Chen Wang for help and grateful discussion.

\section{Authors' contributions}

FJ and LG conceived and designed this study. SX analyzed the data and wrote the manuscript. WM performed experiments and analyzed the data. LX and ZK performed the data analysis. All authors read and approved the manuscript.

\section{Funding}

This work was supported for the design of the study, the data collection, analysis and interpretation and write manuscript by the National Key Research and Development Program (2018YFD1000200), National Nature Science Foundation of China (31872047), The High-level Scientific Research Foundation of Qingdao Agricultural University (665/1119002).

\section{Availability of data and materials}

All data generated or analyzed during this study are included in this published article and in its supplementary information files. The materials are available upon request by contacting the corresponding author. Accession numbers of genes in this study: VVAP2 (MT912541), NtAP2 (MT912542). The VvmiR172 sequence data could also available from the miRBase (http://www.mirbase.org/ summary.shtml?org=vvi) (VvmiR172 accession number: Ml0006544, VvmiR172b 
accession number: MI0006545, VvmiR172C accession number: MI000546, VvmiR172d accession number: MI0006547).

\section{Ethics approval and consent to participate}

Not applicable.

\section{Consent for publication}

Not applicable.

\section{Competing interests}

The authors declare that they have no competing interests.

\section{Author details}

'College of Horticulture, Qingdao Agricultural University, Qingdao 266109, China. ${ }^{2}$ College of Horticulture, Nanjing Agricultural University, Nanjing 210095, China. ${ }^{3}$ Institute of Grape Science and Engineering, College of Horticulture, Qingdao Agricultural University, Qingdao 266109, China.

\section{Received: 8 December 2019 Accepted: 27 August 2020}

Published online: 03 September 2020

\section{References}

1. Wu G. Plant microRNAs and development. J Genet Genomic. 2013;40:217-30

2. Choi K, Kim J, Müller SY, Oh M, Underwood C, Hendersion I, Lee I. Regulation of microRNA-mediated developmental changes by the SWR1 chromatin remodeling complex in Arabidopsis thaliana. Plant Physiol. 2016; 171:1128-43.

3. Ferdous J, Sanchez-Ferrero JC, Langridge P, Milne L, Chowdhury J, Brien C, Tricker PJ. Differential expression of microRNAs and potential targets under drought stress in barley. Plant Cell Environ. 2017;40:11-24.

4. Huang W, Peng S, Xian Z, Lin D, Hu G, Yang L, Ren M, Li Z. Overexpression of a tomato miR171 target gene SIGRAS24 impacts multiple agronomical traits via regulating gibberellin and auxin homeostasis. Plant Biotech J. 2017; 15:472-88

5. Wang M, Sun X, Wang C, Cui L, Chen L, Zhang C, Shangguan LF, Fang J. Characterization of miR061 and its target genes in grapevine responding to exogenous gibberellic acid. Funct Integr Genomics. 2017;17:537-49.

6. Meng Y, Huang F, Shi Q, Cao J, Chen D, Zhang J, Ni J, Wu P, Chen M. Genome-wide survey of rice microRNAs and microRNA-target pairs in the root of a novel auxin-resistant mutant. Planta. 2009;230:883-98.

7. Liu $Q$, Chen $Y Q$. A new mechanism in plant engineering: the potential roles of microRNAs in molecular breeding for crop improvement. Biotechnol Adv. 2010;28:301-7

8. Pantaleo V, Szittya G, Moxon S, Miozzi L, Moulton V, Dalmay T, Burgyan J. Identification of grapevine microRNAs and their targets using highthroughput sequencing and degradome analysis. Plant J. 2010;62:960-76.

9. Liu H, Qin C, Chen Z, Zuo T, Yang X, Zhou H, Xu M, Cao S, Shen Y, Liu H, He $X$, Zhang $Y$, Li L, Ding H, Lübberstedt T, Zhang Z, Pan G. Identification of miRNAs and their target genes in developing maize ears by combined small RNA and degradome sequencing. BMC Genomics. 2014;15:25.

10. Cheng HY, Wang Y, Tao X, Fan YF, Dai Y, Yang H, Ma XR. Genomic profiling of exogenous abscisic acid-responsive microRNAs in tomato (Solanumlycopersicum). BMC Genomics. 2016;17:423.

11. Neilson JR, Zheng GX, Burge CB, Sharp PA. Dynamic regulation of miRNA expression in ordered cellular development. Genes Dev. 2007;21:578-89.

12. Zhang LM, Hammell BA, Kudlow V, Han M. Systematic analysis of dynamic miRNA-target interactions during C. elegans development. Development. 2009;136:3043-55.

13. Jagadeeswaran GY, Zheng N, Sumathipala H, Jiang EL, Arrese JL, Soulages W, Zhang R. Deep sequencing of small RNA libraries reveals dynamic regulation of conserved and novel microRNAs and microRNA-stars during silkworm development. BMC Genomics. 2010;11:52.

14. Schack DV, Agostino MJ, Murray BS, Li Y, Reddy PS, Chen J, Choe SE, Strassle BW, Li C, Bates B, Zhang L, Hu H, Kotnis S, Bingham B, Liu W, Whiteside G, Samad TA, Kennedy JD, Ajit S. Dynamic changes in the microRNA expression pro le reveal multiple regulatory mechanisms in the spinal nerve ligation model of neuropathic pain. PLoS One. 2011;6:E17670.

15. Kirigin FF, Lindstedt $K$, Sellars M, Ciofani M, Low SL, Jones L, Bell F, Pauli F, Bonneau R, Myers RM, Littman DR, Chong MM. Dynamic microRNA gene transcription and processing during T cell development. J Immunol. 2012; 188:3257-67.
16. Wang C, Leng XP, Zhang Y, Kayesh E, Zhang Y, Sun X, Fang JG. Transcriptome-wide analysis of dynamic variations in regulation modes of grapevine microRNAs on their target genes during grapevine development. Plant Mol Biol. 2014:84:269-85.

17. Pang MX, Woodward AW, Agarwal V, Guan X, Ha M, Ramach-andran V, Chen X, Triplett BA, DStelly DM, Chen ZJ. Genome-wide analysis reveals rapid and dynamic changes in miRNA and siRNA sequence and expression during ovule and fiber development in allotetraploid cotton (GossypiumhirsutumL.). Genome Biol. 2009:10:R122.

18. Leng X, Fang J, Pervaiz T, Li Y, Wang X, Liu D, Zhu X, Fang J. Characterization of expression patterns of grapevine microRNA family members using microRNA rapid amplification of complementary DNA ends. Plant Genome. 2015:8(2):1-9.

19. Sieber P, Wellmer F, Gheyselinck J, Riechmann JL, Meyerowitz EM. Redundancy and specialization among plant microRNAs: role of the MIR164 family in developmental robustness. Development. 2007;134:1051-60.

20. Zhao B, Ge L, Liang R, Li W, Ruan K, Lin H, Jin Y. Members of miR169 family are induced by high salinity and transiently inhibit the NF-YA transcription factor. BMC Mol Biol. 2009:10:29.

21. Cui L, Shan JX, Shi M, Gao JP, Lin HX. The miR156-SPL9-DFR pathway coordinates the relationship between development and abiotic stress tolerance in plants. Plant J. 2014;80:1108-17.

22. Velasco R, Zharkikh A, Troggio M, Cartwright DA, Cestaro C. A high quality draft consensus sequence of the genome of a heterozygous grapevine variety. PLoS One. 2007;2:E1326.

23. Wang C, Shangguan LF, Korir NK, Wang XC, Han J, Song CN, Fang JG. Characterization of microRNAs identified in a table grapevine cultivar with validation of computationally predicted grapevine miRNAs by miR-RACE. PLoS One. 2011;6:E21259.

24. Wang C, Wang XC, Kibet NK, Song CN, Zhang C, Li XY, Han J, Fang JG. Deep sequencing of grapevine flower and berry short RNA library for discovery of novel microRNAs and validation of precise sequences of grapevine microRNAs deposited in miRBase. Physiol Planturm. 2011;143:64-81.

25. Song C, Jia Q, Fang J, Li F, Wang C, Zhang Z. Computational identification of citrus microRNAs and targets analysis in citrus expressed sequence tags. Plant Biol. 2010:12:927-34.

26. Sun X, Zhang Y, Zhu X, Korir NK, Tao R, Wang C, Fang J. Advances in identification and validation of plant microRNAs and their targets genes. Physiol Plantarum. 2014;152:203-18.

27. Zhao B, Liang R, Ge L, Li W, Xiao H, Lin H, Ruan K, Jin Y. Identification of drought-induced microRNAs in rice. Biochem Biophys Res Commun. 2007 354:585-90.

28. Wu G, Park MY, Conway SR, Wang JW, Weigel D, Poethig RS. The sequential action of miR156 and miR172 regulates developmental timing in Arabidopsis. Cell. 2009:138:750-9.

29. Zhu $\mathrm{QH}$, Helliwell CA. Regulation of flowering time and floral patterning by miR172. J Exp Bot. 2011:62:487-95.

30. Wang CY, Zhang S, Yu Y, Luo YC, Liu Q, Ju C, Zhang YC, Qu LH, Lucas WJ, Wang C, Chen YQ. MiR397b regulates both lignin content and seed number in Arabidopsis via modulating a laccase involved in lignin biosynthesis. Plant Biotechnol J. 2014;12:1132-42.

31. Sun Z, Su C, Yun J, Jiang Q, Wang L, Wang Y, Cao D, Zhao F, Zhao Q, Zhang M, Zhou B, Zhang L, Kong F, Liu B, Tong Y, Li X. Genetic improvement of the shoot architecture and yield in soybean plants via the manipulation of GmmiR156b. Plant Biotech J. 2019;17:50-62.

32. Sharma D, Tiwari M, Pandey A, Bhatia C, Sharma A, Trivedi PK. MicroRNA858 is a potential regulator of phenylpropanoid pathway and plant development in Arabidopsis. Plant Physiol. 2016;171:944-74.

33. Liu J, Cheng X, Liu P, Sun J. miR156-targeted SBP-box transcription factors interact with DWARF53 to regulate TEOSINTE BRANCHED1 and BARREN STALK1 expression in bread wheat. Plant Physiol. 2017;174:1931-48.

34. Nguyen STT, Greaves T, McCurdy DW. Heteroblastic development of transfer cells is controlled by the microRNA miR156/SPL module. Plant Physiol. 2017; 173:1676-91.

35. Wang C, Han J, Korir NK, Wang X, Liu H, Li X, Leng X, Fang J. Characterization of target mRNAs for grapevine microRNAs with an integrated strategy of modified RLM-RACE, newly developed PPM-RACE and qPCRs. J Plant Phyisol. 2013;170:943-57.

36. Nova-Franco B, Iniguez LP, Valdes-Lopez O, Alvarado-Affantranger X, Leija A, Fuentes SI, Ramirez M, Paul S, Reyes JL, Girard L, Hernandez G. Themicro- 
RNA72C-APETALA2-1 node as a key regulator of the common bean-

Rhizobium etli nitrogen fixation symbiosis. Plant Physiol. 2015;168:273-91.

37. Chen XM. A MicroRNA as a translational repressor of APETALA2 in Arabidopsis flower development. Science. 2004;303:2022-5.

38. François $L$, Verdenaud M, Fu X, Ruleman D, Dubois A, Vandenbussche $M$, Bendahmane A, Raymond O, Just J, Bendahmane M. A miR172 targetdeficient AP2-like gene correlates with the double flower phenotype in roses. Sci Rep-UK. 2018;8:12912.

39. Tang M, Bai X, Niu LJ, Chai X, Chen MS, Xu FZ. miR172 regulates both vegetative and reproductive development in the perennial woody plant Jatropha curcas. Plant Cell Physiol. 2018:59:2549-63.

40. Lee I, Lee J. Regulation and function of SOC1, a flowering pathway integrator. J Exp Bot. 2010;61:2247-54

41. Jung HJ, Seo YH, Seo PJ, Reyes JL, Yun J, Chua NH, Park CM. The GIGANTEAregulated microRNA172 mediates photoperiodic flowering independent of CONSTANS in Arabidopsis. Plant Cell. 2007;19:2736-48.

42. Ioannidi E, Rigas S, Tsitsekian D, Daras G, Alatzas A, Makris A, Tanou G, Argiriou A, Aexandrou D, Poethig S, Hatzopoulos P, Kanellis AK. Trichome patterning control involves TTG1 interaction with SPL transcription factors. Plant Mol Biol. 2016;92:675-87.

43. Shi R, Chiang VL. Facile means for quantifying microRNA expression by realtime PCR. Biotechniques. 2005:39:519-25.

44. Moxon S, Jing R, Szittya G, Schwach F, Pilcher RL, Moulton V, et al. Deep sequencing of tomato short RNAs identifies microRNAs targeting genes involved in fruit ripening. Genome Res. 2008;18:1602-160.

45. Mlotshwa S, Verver J, Sithole-Niang I, Prins M, Van Kammen AB, Wellink J. Transgenic plants expressing HC-Pro show enhanced virus sensitivity while silencing of the transgene results in resistance. Virus Genes. 2002;25:45-57.

\section{Publisher's Note}

Springer Nature remains neutral with regard to jurisdictional claims in published maps and institutional affiliations.

Ready to submit your research? Choose BMC and benefit from:

- fast, convenient online submission

- thorough peer review by experienced researchers in your field

- rapid publication on acceptance

- support for research data, including large and complex data types

- gold Open Access which fosters wider collaboration and increased citations

- maximum visibility for your research: over $100 \mathrm{M}$ website views per year

At $\mathrm{BMC}$, research is always in progress.

Learn more biomedcentral.com/submissions 